\title{
Hypothesis theory and the PREE
}

\author{
JUDITH TADDONIO and MARVIN LEVINE \\ State University of New York at Stony Brook, Stony Brook, New York 11794
}

\begin{abstract}
A recent expansion of hypothesis theory has resulted in a new interpretation of the partial-reinforcement extinction effect (PREE). According to this interpretation, the classical 100\%-reinforcement and 50\%-reinforcement groups receive different solution-experiences on Problem 1. This, in turn, produces different expectations about the solution on Problem 2. Three experiments, using a nonreversal shift paradigm, were performed to test this view. The results were that, as hypothesized, a nonreversal shift was learned faster than a shift from a random-reinforcement condition.
\end{abstract}

Hypothesis $(\mathrm{H})$ theory provides a useful description of the behavior of human subjects during discrimination-learning tasks (Bower \& Trabasso, 1963; Levine, 1966). Traditionally, the theory has emphasized within-problem dynamics, i.e., the process of selecting and rejecting hypotheses in order to arrive at the solution to a particular problem. Recently, however, Levine (1974) has addressed himself to the problem of transfer, i.e., to the changes which occur across a series of problems. Levine proposed that the solutions experienced on the early problems suggest to the subject the class of solutions for the entire series. The subject, for example, who receives a series of problems with position-sequence solutions will soon recognize the similarity of the solutions from problem to problem and will then start each new problem looking for sequence solutions. This conception resulted in an expansion of $\mathrm{H}$ theory. Specifically, the following "transfer hypothesis" was added to the existing theory:

When a subject receives a series of problems, he infers from the first $n$ solutions the domain within the universe from which the $n+1$ st solution may be taken. He starts the $n+1$ st problem by sampling hypotheses from that domain. Hypothesis theory, then, views transfer as dependent upon the type of solution experience the subject has had. This experience predisposes the subject toward a particular domain of hypotheses from which he believes subsequent solutions will be drawn.

Levine (1974) has discussed implications of this conception for several standard phenomena, one of which is the partial-reinforcement extinction effect (PREE-see Humphreys, 1939; Meyers, Dreissen, \& Halpern, 1972). In one version of the PREE paradigm subjects are asked to predict which of two panels will light up by making an appropriate prediction response (e.g., touching one of the two panels). The subjects receive a long series of prediction trials under one of two

This research was supported by Grant $M H 11857$ from the National Institute of Mental Health awarded to Marvin Levine. The authors would like to express their appreciation to Irwin Jankovic for his assistance with Experiment III.

Requests for reprints should be sent to Marvin Levine, Department of Psychology, State University of New York at Stony Brook, Stony Brook, New York 11790. conditions: In the $100 \%$ condition the same panel illuminates at every trial; in the $50 \%$ condition, the two panels light up randomly. This set of trials is followed by a second set which, for both conditions, consists of lighting on every trial the panel that, for the $100 \%$ group, had never come on. The classical finding is that the $100 \%$ group "extinguishes" faster than the 50\% group, i.e., that the $100 \%$ group requires fewer trials to be consistently correct during the second phase.

The traditional interpretation of this task is that it consists of two phases. The $100 \%$ group is "conditioned" to make one response in Phase 1. In Phase 2 this response is "extinguished", and the other response is newly conditioned. For the $50 \%$ group, no conditioning (i.e., no strengthening of either response) occurs in Phase 1. However, the 50\% group takes longer to condition in Phase 2, i.e., it shows slower extinction.

According to the present approach, the subject is presented not with a single sequence of trials divided into two phases, but with two problems. From this perspective, the two groups differ on Problem 2 because of differing solution experience on Problem 1. Subjects in the $100 \%$ group have experienced a problem with a simple solution, and should tend to begin Problem 2 (i.e., so-called extinction) by searching within the domain of simple hypotheses for the solution to the problem. Since the solution to the second problem is drawn from that domain, subjects in the $100 \%$ group should, on the average, solve the problem quickly. Subjects in the $50 \%$ group, however, do not experience a simple solution on Problem 1, and are likely to begin Problem 2 by searching within a more complex domain for solution. This search should hinder their performance on Problem 2.

The transfer hypothesis thus provides a plausible explanation of the PREE. It also has relevance to a variation on this phenomenon. The traditional PREE task has a single dimension, position. Problem 2 represents a reversal shift for the $100 \%$ group. Suppose, however, that a two-dimensional task were employed. For the experiment described above, imagine that the two panels were each of two colors and that these colors were randomly assigned to the two panels from trial to 
trial. A nonreversal shift from Problem 1 to Problem 2 may now be presented. Suppose, that is, that for the $100 \%$ group the left side were correct on Problem 1 and that the choice of the color red were correct on Problem 2. The 50\% group would parallel the $100 \%$ group in the same fashion as before: Feedback would be contingent on neither location nor color during the first problem, and choice of the color red would be correct on Problem 2. From the standpoint of traditional considerations, during Problem 1, response to each color is intermittently reinforced for both groups. It is not clear, therefore, that the $100 \%$ group should solve the second problem faster than the $50 \%$ group. According to the transfer hypothesis, however, the $100 \%$ group should have an advantage. Again, these subjects experience a simple solution on Problem 1. They will, therefore, tend to begin Problem 2 by testing Hs about simple solutions. Subjects in the $50 \%$ group, on the other hand, should tend to begin Problem 2 by searching within a domain of complex hypotheses. The transfer hypothesis, then, predicts that a nonreversal shift will also be learned faster than a shift from a random condition. The present experiments were performed to check this implication.

\section{METHOD}

Three experiments, all having the same basic paradigm, were performed. Only the methodology of Experiment I will be outined in detail. The variations instituted in Experiments II and III will be listed separately following the description of Experiment I.

\section{Experiment I}

Subjects. The subjects were 48 undergraduates from the State University of New York at Stony Brook who were paid for their participation.

Materials. Stimulus materials were constructed from $4 \times 6$ in. white index cards. The letters " $A$ " and " $B$ " were printed on each card, with one letter appearing within a box on the left side of the card and the other appearing within a box on the right side of the card. The boxes were1-1/2-in. tall and 1-in. wide, with a 1-1/2-in. space between the two boxes. One hundred such cards were arranged into a stimulus deck: For half the cards, the letter "A" was on the left side, for the other half, "B" was on the left. The two letters varied randomly from side to side within the deck with the restriction that letter and side were not confounded more than three times in a row. A card printed with the words "please wait" was placed on the top of the deck.

Design and procedure. All subjects were instructed that they would be shown a series of cards made up of "As" and "Bs" and that these letters would appear within a box on the left or right side of the card. For every card they were asked to indicate verbally which box (i.e., left or right) was the correct choice for that card. The subjects were informed that after they had made a response the experimenter would indicate whether they had been correct or wrong; they were then to turn to the next card and make their next response.

The subjects were assigned to one of two groups:

$100 \%$ Group. These subjects received an initial discrimination-learning problem, the solution to which was drawn from the four simple, univalued alternatives: Choose the box containing "A," choose the box containing "B," choose the box on the left, choose the box on the right. The four solutions were counterbalanced across subjects. The problem lasted until the subject reached either a criterion of 15 correct responses in a row, or a total of 40 trials. At that point, Problem 2 was begun. Problem 2 constituted a nonreversal shift of solution (e.g., left side on Problem 1 and " $A$ " on Problem 2). The eight possible shifts were counterbalanced over the 24 subjects.

$50 \%$ Group. Prior to the experimental session, the experimenter drew up an irregular correct-wrong sequence. This sequence had the constraint that none of the four simple hypotheses could be confirmed from more than three consecutive trials. This random sequence was applied to the responses of these subjects during Problem 1. Each subject in the $50 \%$ group was matched to a subject in the $100 \%$ group according to two characteristics: He received both the same number of trials as that $100 \%$ subject and the same solution on Problem 2.

All subjects were run to a criterion of 15 consecutive correct responses, or to a total of 60 trials on Problem 2.

\section{Experiment II}

The subjects in the $100 \%$ group not only experience a typical solution on Problem 1, but also have another potential advantage. They receive a signal announcing the start of Problem 2: After saying "correct" for 15 trials in a row, the experimenter suddenly says "wrong." A comparable feedback pattern is, of course, not available to the subjects in the $50 \%$ group. The two groups could be rendered more equivalent in this respect by providing both with an explicit signal. This was instituted in the present experiment. Experiment II, then, was identical to Experiment I, except that a procedural break was instituted between the two problems. The break took the form of the following instructions to the subjects: "Fine, that's the end of the first problem. I'd like you to try another, beginning with the next card."

\section{Experiment III}

In contrast to the simultaneous paradigm used in Experiments I and II, Experiment III employed a paradigm of successive discrimination for the two problems. Subjects in Experiment III viewed single letters (" $A$ " or " $B$ ") which were printed in red or black ink on index cards. The four letter-color combinations were counterbalanced within the deck of stimulus cards, again with the constraint that the same card not appear more than three times in a row. The subjects were instructed to sort the cards into two groups by saying "left" or "right" to each card. In all other details, Experiment III paralleled that of Experiment II.

\section{RESULTS AND DISCUSSION}

The performance of subjects in the $50 \%$ group was inferior to that of the $100 \%$ group in each of the three experiments. Inspection of Table 1 reveals that subjects in the $50 \%$ group took almost twice the number of trials to solve the problem as did subjects in the $100 \%$ group. Some hint of the generality of the result is evident: The difference held even when both groups were signaled that the contingencies had changed, and appeared in both a simultaneous- and a successive-discrimination task.

The transfer hypothesis provides a reasonable explanation of these results. This approach describes the differences between the $50 \%$ and $100 \%$ groups in terms of differential solution experience on Problem 1. Subjects in the 50\% group received a difficult first problem which predisposed them to search within a 
Table 1

Mean Trials to Criterion on the Second Problem

\begin{tabular}{ccc}
\hline Experiment & $100 \%$ Group & $50 \%$ Group \\
\hline 1 & 10.7 & $17.8^{*}$ \\
2 & 7.6 & $16.8^{* *}$ \\
3 & 19.6 & $34.0^{* *}$ \\
\hline
\end{tabular}

*Not significant

domain of complex hypotheses for the solution to Problem 2. Subjects in the $100 \%$ group received a simple first problem, which tended to direct them to search for simple solutions to the next problem. Since the solution to Problem 2 was, in fact, from the simple domain, the $100 \%$ group solved relatively quickly, whereas the $50 \%$ group was hindered.

\section{REFERENCES}

Bower, G., \& Trabasso, T. Concept Identification. In R. C Atkinson (Ed.), Studies in mathematical psychology. Stanford: Stanford University Press, 1963.

Humphreys, L. G. Acquisition and extinction of verbal expectation in a situation analagous to conditioning. Journal of Experimental Psychology, 1939, 25, 284-301.

Levine, M. Hypothesis behavior by humans during discrimination learning. Journal of Experimental Psychology, 1966, 71, 331-338.

Levine, M. A transfer hypothesis, whereby learning-to-learn, Einstellung, the PREE, reversal-nonreversal shifts, and other curiosities are elucidated. In R. L. Solso (Ed.) Theories in cognitive psychology; The Loyola symposium, Lawrence Erlbaum Associates, Potomac, Maryland, 1974.

Meyers, L. S., Dreissen, E., \& Halpern, J. Transfer following regular and irregular sequences of events in a guessing situation. Journal of Experimental Psychology, 1972, 92, 182-190.

(Received for publication October 1, 1974.)

Bulletin of the Psychonomic Society

1975, Vol. 5 (1), 76-78

\title{
Resistance to extinction in humans as a function of sequential variables and amount of acquisition training
}

\author{
R. SCHMECK and C. MUELLER \\ Southern Illinois University, Carbondale, Illinois 62901
}

\begin{abstract}
The experimenters used human subjects and three schedules of reinforcement: (1) continuous reinforcement; (2) a schedule with few N-R transitions and long N-lengths; and (3) a schedule with many N-R transitions and short N-lengths. In the first experiment, with extended acquisition training, the second schedule produced a partial reinforcement effect, but the third did not. In the second experiment, acquisition training was shortened, but the results were essentially the same as those of the first experiment.
\end{abstract}

Since the early demonstrations of partial reinforcement effects in human subjects (Humphreys, $1939 a, 1939 b)$, there have been relatively few studies investigating the effects of sequential variables on the phenomenon. Grosslight, Hall, and Murnin (1953) and Poon and Halpern (1971) demonstrated that a partial reinforcement schedule produces greater resistance to extinction in human subjects if it contains more trials on which the response is rewarded immediately following trials on which the response is not rewarded (N-R transitions). In addition, Meyers and Capaldi (1970) and Halpern and Poon (1971) determined that the resistance to extinction of human subjects was increased if they received more consecutive nonrewarded trials prior to rewarded trials (N-length) (i.e., schedules which gave subjects more experience with longer N-lengths produced greater resistance to extinction).

When studying the effect of N-R transitions and $\mathrm{N}$-length, one encounters a problem inherent in attempting to vary one sequential variable while holding the other constant. Variation in one of the variables tends to produce simultaneous variation in the other.
Grosslight et al. (1953) attempted to vary N-R transitions while holding $\mathrm{N}$-length constant by giving the group that was to receive no $\mathrm{N}-\mathrm{R}$ transitions a brief rest period following each series of nonrewarded trials. However, the authors point out that these subjects might well have retained the experience of nonreward during the rest period. Thus, they would have had the subjective experience of an N-R transition when the reinforcement schedule was resumed following the rest period.

The present studies varied N-R transitions and N-lengths simultaneously. Capldi (1967) has indicated that, when rats are used as subjects, the impact of sequential variables is related to the number of training trials given prior to extinction. When subjects are given relatively extended training (i.e., when asymptotic habit strength is reached), N-length is more frequently the major determiner of resistance to extinction. This prediction was supported by Halpern and Poon (1971) using extended training, but no continuous reinforcement control group was included. The first study reported below gave subjects extended training 